\title{
The Microbiology of Diabetic Foot Ulcer Infections - Prospective Study
}

\author{
K. Sajitha* \\ Department of Microbiology,, Dr B R Ambedkar Medical College, Bengaluru, India \\ *Corresponding author
}

Keywords

Diabetic foot ulcer, Staphylococcus aureus,

Enterococcus faecalis

Article Info

Accepted:

04 April 2019

Available Online:

10 May 2019
A B S T R A C T

Diabetic foot ulcer is a major complication of diabetes mellitus, and probably the major component of the diabetic foot. Wound healing is an innate mechanism of action that works reliably most of the time. The present study was carried out in Department of Microbiology, in a tertiary care hospital, over a period of one year from June 2016 - May 2017. 50 diabetic patients with foot ulcer attending the Surgery Out Patient Department were included in the study. These patients were diabetic or detected to be diabetic by blood glucose estimation at the time of their visit to the hospital. In the present study, Of the 50 cases studied, most of the patients belonged to $>60$ years of life. Males were more affected compared to females with a ratio 1.6:1. Among Gram positive aerobes, Staphylococcus aureus was the predominant isolate (23.5\%) followed by Enterococcus faecalis in $9.4 \%$ of cases. Among Gram negative aerobes, Proteus mirabilis was the most common isolate (19.8\%) and followed by Pseudomonas aeruginosa and Klebsiella pneumoniae in $9.4 \%$ of the cases.

\section{Introduction}

Diabetic foot ulcer is a major complication of diabetes mellitus, and probably the major component of the diabetic foot. Wound healing is an innate mechanism of action that works reliably most of the time. A key feature of wound healing is stepwise repair of lost extracellular matrix (ECM) that forms the largest component of the dermal skin layer. ${ }^{1}$ But in some cases, certain disorders or physiological insult disturbs the wound healing process. Diabetes mellitus is one such metabolic disorder that impedes the normal steps of the wound healing process. Many studies show a prolonged inflammatory phase in diabetic wounds, which causes a delay in the formation of mature granulation tissue and a parallel reduction in wound tensile strength. ${ }^{2}$ Treatment of diabetic foot ulcers should include: blood sugar control, removal of dead tissue from the wound, wound dressings, and removing pressure from the wound through techniques such as total contact casting. Surgery in some cases may improve outcomes. ${ }^{3}$ Hyperbaric oxygen therapy may also help but is expensive. ${ }^{3}$ It occurs in $15 \%$ of people with diabetes, ${ }^{4}$ and precedes $84 \%$ of all diabetes-related lower-leg amputations. $^{5}$

Skin commensals such as coagulase-negative Staphylococci, Corynebacterium, or Micrococcus from swab cultures are not 
usually considered true pathogens, although they may grow repeatedly or from reliable specimens. In most centers, including Korea, $S$. aureus is the most frequently isolated, and perhaps most virulent pathogen, whether alone or in combination. Aerobic Grampositive cocci, especially $S$. aureus and Streptococcus species, are the predominant pathogens in DFIs and usually cause monomicrobial infection in previously untreated acute infections. Polymicrobial infections, which may include various types of aerobes such as S. aureus, Streptococcus, Enterococcus, Enterobacteriaceae, and Pseudomonas commonly, appear in deep or chronic wounds. Polymicrobial infections caused by multi-drug resistant, aerobic Gramnegatives predominated in a tertiary care center in India and Pakistan. In contrast to Western countries, $S$. aureus is less prevalent and $P$. aeruginosa is more common in developing countries with warm climates, especially Asia and Africa. The reasons for this are not clear but may be related to environmental factors, footwear, personal hygiene, antimicrobial pre-treatment, or other factors. 6,7

In Korea, Gram-positive aerobes such as Staphylococcus, Streptococcus, and Enterococcus are more common than Gramnegative aerobes such as Enterobacteriaceae and Pseudomonas. Among them, S. aureus is the most common pathogen, with a prevalence ranging from $26.2 \%$ to $46.3 \%$. Anaerobes are predominantly seen in DFIs with ulcers that are deeper and more chronic and are associated with ischemia, necrosis, gangrene, or foul odor; however, their clinical significance is not yet clear. Genetic (molecular) analysis can rapidly and reliably detect many more microorganisms (especially anaerobes) than conventional culture methods and is used for characterization, determination of virulence, and the potential antibiotic resistance of pathogens in patients with DFIs.
Their role in improving the clinical care of patients with DFIs will become more significant with the use of metagenomics, transcriptomics, proteomics, and metabolomics. $^{8,9}$

\section{Objective}

To isolate the bacteria responsible for infection in diabetic foot ulcers

\section{Materials and Methods}

\section{Source of data}

The present study was carried out in Department of Microbiology, DR B R AMBEDKAR MEDICAL COLLEGE, BENGALURU, over a period of one year from June 2016 - May 2017. 50 diabetic patients with foot ulcer attending the Surgery Out Patient Department were included in the study. These patients were diabetic or detected to be diabetic by blood glucose estimation at the time of their visit to the hospital.

\section{Inclusion criteria}

Type I and II diabetes of all the age groups and both sexes.

Foot ulcers of Grade I or more.

\section{Exclusion criteria}

Patients on antibiotic treatment.

Foot ulcers of Grade 0.

\section{Results and Discussion}

Of the 50 cases studied, most of the patients belonged to $>60$ years of life. Males were more affected compared to females with a ratio 1.6:1. Among Gram positive aerobes, Staphylococcus aureus was the predominant isolate $(23.5 \%)$ followed by Enterococcus 
faecalis in $9.4 \%$ of cases. Among Gram negative aerobes, Proteus mirabilis was the most common isolate (19.8\%) and followed by Pseudomonas aeruginosa and Klebsiella pneumoniae in $9.4 \%$ of the cases.

In the present study, Of the 50 cases studied, most of the patients belonged to $>60$ years of life. Males were more affected compared to females with a ratio 1.6:1. Among Gram positive aerobes, Staphylococcus aureus was the predominant isolate $(23.5 \%)$ followed by Enterococcus faecalis in $9.4 \%$ of cases. Among Gram negative aerobes, Proteus mirabilis was the most common isolate (19.8\%) and followed by Pseudomonas aeruginosa and Klebsiella pneumoniae in $9.4 \%$ of the cases.

Louie TJ et al., (1976) ${ }^{10}$ studied 20 diabetic foot ulcers. Specimens were obtained by scraping ulcer base or a deep portion of wound edge after debridement of superficial exudates. The specimens were inoculated at bedside onto aerobic and anaerobic culture media. There were a total of 116 isolates of which 64 were aerobes and 52 were anaerobes with an average of 5.8 bacterial species per specimen. 35 Gram positive bacteria; predominantly Streptococcus faecalis \& 29 Gram negative bacteria; predominantly Proteus species were isolated in their study.
Major anaerobes were Peptococcus species and Bacteroides fragilis. All had polymicrobial infection except one which had pure growth of Clostridium perfringens.

Charles S Sharp et al., (1979) ${ }^{11}$ studied 58 superficial \& deep ulcers. In 10 of the 58 superficial and deep double specimens, identical organisms or combinations of organisms were found at both sites. No common organisms in both the sites were seen in 13 patients whereas in 9 patients, all the organisms found in initial superficial cultures were also found in deep specimen with additional organisms in deep specimens. In 8 patients, organisms isolated from deep and superficial cultures were same whereas in 18 patients they isolated different organisms from different sites.

The most common organisms isolated from both sites were Proteus species and Enterococci. Most frequent anaerobe isolated was Bacteroides fragilis. Bamberger DM et al $(1987)^{27}$ studied 51 diabetic patients with osteomyelitis of foot. 153 organisms were isolated. Staphylococcus aureus along with Group D Streptococci were the commonest. Gram positive cocci accounted for nearly $48 \%$ of the isolates (Table 1 and 2).

Table.1 Age and sex wise distribution of cases

\begin{tabular}{|l|l|l|l|l|l|c|}
\hline \multirow{2}{*}{$\mathbf{S}$} & \multicolumn{2}{|c|}{ Male } & \multicolumn{2}{c|}{ Female } & \multicolumn{2}{c|}{ Total } \\
\cline { 2 - 6 } & No. & $\%$ & No. & $\%$ & No. & $\%$ \\
\hline 31-40 years & 01 & 2 & 01 & 2 & 02 & 04 \\
\hline 41-50 years & 05 & 10 & 01 & 2 & 06 & 12 \\
\hline $51-60$ years & 11 & 22 & 06 & 12 & 17 & 34 \\
\hline$>60$ years & 14 & 28 & 11 & 22 & 25 & 50 \\
\hline Total & 31 & 62 & 19 & 38 & 50 & 100 \\
\hline
\end{tabular}


Table.2 Number and percentage of aerobes isolated

\begin{tabular}{|c|c|c|}
\hline GRAM POSITIVES & No. of aerobes $(\mathbf{n = 1 0 6})$ & Percentage $(\%)$ \\
\hline Staphylococcus aureus & 25 & $\mathbf{2 3 . 5}$ \\
\hline Enterococcus faecalis & 10 & $\mathbf{9 . 4}$ \\
\hline $\begin{array}{c}\text { Coagulase negative } \\
\text { Staphylococcus } \text { (CONS) }\end{array}$ & 05 & $\mathbf{4 . 7}$ \\
\hline Group A Streptococci & 02 & $\mathbf{1 . 8}$ \\
\hline Corynebacterium species & 01 & $\mathbf{0 . 9}$ \\
\hline GRAM NEGATIVES & & \\
\hline Proteus mirabilis & 21 & $\mathbf{1 9 . 8}$ \\
\hline Pseudomonas aeruginosa & 10 & $\mathbf{9 . 4}$ \\
\hline Klebsiella pneumoniae & 10 & $\mathbf{9 . 4}$ \\
\hline Escherichia coli & 9 & $\mathbf{8 . 5}$ \\
\hline Proteus vulgaris & 4 & $\mathbf{3 . 7}$ \\
\hline Klebsiella oxytoca & 3 & $\mathbf{2 . 8}$ \\
\hline Citrobacter freundii & 1 & $\mathbf{0 . 9}$ \\
\hline Acinetobacter species & 3 & $\mathbf{2 . 8}$ \\
\hline Enterobacter species & $\mathbf{3}$ & $\mathbf{2 . 8}$ \\
\hline
\end{tabular}

Gram negative bacilli accounted for nearly $20 \%$ of the isolates, predominated by Enterobacter cloacae, Proteus species, Pseudomonas aeruginosa and Klebsiella pneumoniae. $18 \%$ of the isolates were obligate anaerobes among which Peptococcus species was commonest followed by Bacteroides species. Clostridium species constituted $2 \%$ of the isolates.

Ramani et al., $(1991)^{12}$ studied 75 diabetic foot ulcers of grade 2 or more. A total of 223 isolates (162 aerobes and 61 anaerobes) with an average of 2.97 bacteria per specimen were isolated. Staphylococcus aureus was the predominant isolate recovered in $60 \%$ of cases.

The other aerobic organisms isolated were Proteus mirabilis (26.67\%), Klebsiella species (26.67\%), Pseudomonas species $(25.33 \%)$. Anaerobic bacteria were isolated in 46 patients (61.33\%). These included Bacteroides fragilis (32\%), Peptococcus (17.33\%) and Clostridium welchii (10.67\%).
Grayson et al., (1994) ${ }^{13}$ studied 92 diabetic patients with limb threatening foot infections. Polymicrobial nature of infections was noted. Staphylococcus aureus was the commonest isolate. The other Gram positive aerobic cocci isolated were Enterococcus sps, Streptococcus sps and Coagulase negative Staphylococcus. Gram negative facultative aerobes were less frequent \& consisted of Pseudomonas aeruginosa, Escherichia coli, Klebsiella species, Proteus species and others. Amongst anaerobes, Non-fragilis Bacteroides species was commonest.

In conclusion, approximately 15 percent of people with diabetes experience foot ulcers. And approximately 84 percent of lower limb amputations have a history of ulceration with only approximately half of amputees surviving for more than 2 years. 56 percent of individuals with foot ulcers who do not have amputations survive for 5 years. Foot ulcers and amputations significantly reduce the quality of life. Approximately 8.8 percent of hospital admissions of diabetic patients are 
for foot related problems, and such hospital admissions are about 13 days longer than for diabetics without foot related admissions. Approximately 35 to 40 percent of ulcers recur within 3 years and up to 70 percent recur within 5 years. Diabetic foot disease is the leading cause of non-traumatic lower limb amputations.

\section{References}

1. Nomikos Iakovos, N., et al., (2006). "Protective and Damaging Aspects of Healing: A Review". Wounds. 18 (7): 177-185.

2. McLennan, S; et al., (2006). "Molecular aspects of wound healing" (PDF). Primary Intention. 14 (1): 8-13.

3. Yazdanpanah, L, Nasiri, M., Adarvishi, S (15 February 2015). "Literature review on the management of diabetic foot ulcer". World Journal of Diabetes. 6 (1): 37-53. doi: 10.4239/wjd.v6.i1.37.

4. Brem, H., Tomic-Canic, M., (2007). "Cellular and molecular basis of wound healing in diabetes". Journal of Clinical Investigation. 117 (5): 1219-1222.

5. Turns, M., (2013). "Diabetic foot ulcer management: the podiatrist's perspective". $\mathrm{Br} \mathrm{J}$ Community Nurs. Suppl: S14, S16-9.

6. Uckay, I., Gariani K, Pataky Z, Lipsky BA. Diabetic foot infections: state-of-theart. Diabetes Obes Metab. 2014; 16: 305316.

7. Miyan, Z., Fawwad A, Sabir R, Basit A. Microbiological pattern of diabetic foot infections at a tertiary care center in a developing country. J Pak Med Assoc. 2017; 67: 665-669.

8. Lipsky, BA., Aragón-Sánchez J, Diggle M, Embil J, Kono S, Lavery L, Senneville É, Urbančič-Rovan V, Van Asten S. International Working Group on the Diabetic Foot, Peters EJ. IWGDF guidance on the diagnosis and management of foot infections in persons with diabetes. Diabetes Metab Res Rev. 2016; 32(Suppl 1): 45-74.

9. Young, H., Miller W, Burnham R, Heard S, Berg C, Jenkins TC. How do preoperative antibiotics affect culture yield in diabetic foot infections? Open Forum Infect Dis. 2017; 4: ofx016.

10. Louie, TJ., Bartlett JG, Tally FP, Gorbach SL. Aerobic and Anaerobic Bacteria in Diabetic Foot Ulcers. Annals of Internal Medicine, 1976; 85: 461-463.

11. Sharp, CS., Bessman AN, Wagner FW Jr, Garland D, Reece E. "Microbiology of superficial and deep tissues in infected diabetic gangrene." Surg Gynaecol Obstet, 1979, 149: 217-219.

12. Ramani, A., Ramani R, Shivananda PG, Kundaje GN. "Bacteriology of Diabetic Foot Ulcers." Indian J Pathol. Microbiol, 1991; 34(2): 81-87.

13. Grayson, ML., Gibbons GW, Habershaw GM, Freeman DV, Pomposelli FB, Rosenblum BI, Levin E, Karchmer AW. "Use of Ampicillin/ Sulbactam Versus Imipenem/ Cilastatin in the treatment of limb-threatening foot infections in diabetes patients." Clinical Infectious Diseases, May 1994; 18: 683-693.

\section{How to cite this article:}

Sajitha, K. 2019. The Microbiology of Diabetic Foot Ulcer Infections - Prospective Study. Int.J.Curr.Microbiol.App.Sci. 8(05): 274-278. doi: https://doi.org/10.20546/ijcmas.2019.805.031 